EPJ Web of Conferences 64, 07002 (2014)

DOI: $10.1051 /$ epjconf/ 20146407002

(C) Owned by the authors, published by EDP Sciences, 2014

\title{
V2487 Oph 1998: a post nova in an intermediate polar
}

\author{
Margarita Hernanz ${ }^{1, a}$ \\ ${ }^{1}$ Institute of Space Sciences - ICE (CSIC-IEEC), Campus UAB, \\ Fac. Ciències, C5 par $2^{a}$ pl., 08193 Bellaterra (Barcelona), Spain
}

\begin{abstract}
V2487 Oph (Nova Oph 1998) is a classical nova that exploded in 1998. XMM-Newton observations performed between 2 and 9 years after the explosion showed emission related to restablished accretion, and indicative of a magnetic white dwarf. The spectrum looks like that of a cataclysmic variable of the intermediate polar type. Anyway, we don't have yet a definitive confirmation of the intermediate polar character, through determination of spin and orbital periods. Although it is not the first nova exploding in a magnetic white dwarf, it is always challenging to reach explosive conditions when a standard accretion disk can't be formed, because of the magnetic field. In addition, V2487 Oph has been the first nova where a detection of X-rays - in the host binary system - has been reported prior to its eruption, in 1990 with the ROSAT satellite. V2487 Oph has been also detected in hard X-rays with INTEGRAL/IBIS and RXTE/PCA. Last but not least, V2487 Oph has been identified as a recurrent nova in 2008, since a prior eruption in 1900 has been reported through analysis of Harvard photographic plates. Therefore, it is expected to host a massive white dwarf and be a candidate for a type Ia supernova explosion. In a recent study of the progenitors of galactic novae, it has been emphasized that V2487 Oph is an important and interesting object, "intermediate" between the "standard" classical novae and other historical and well-known recurrent novae with shorter recurrence periods. It could be that in the end there's a continuous distribution of recurrence periods, instead of the common understanding up to now that "classical" and "recurrent" novae were quite apart (with recurrence periods of more than $10^{4}$ years and less than 100 years - approximately - respectively). We present the results of our campaign of several observations with XMM-Newton. The consequences for the understanding of such a puzzling object are discussed.
\end{abstract}

\section{Scenarios of nova explosions}

White dwarfs (WDs) can explode as classical novae in two types of binary systems, a cataclysmic variable (the most common case) and a symbiotic binary. The companion star is a main sequence star, in the first case, and a red giant in the second. Accretion in cataclysmic variables happens via Roche lobe overflow of the secondary, companion star, towards the white dwarf, whereas a stellar wind (and also an accretion disk in some cases) is responsible for accretion onto white dwarfs in symbiotic binaries. The orbital periods of CVs range between 10 minutes and 10 hours approximately, whereas they are much longer in symbiotics (100's of days).

\footnotetext{
a e-mail: hernanz@ieec.uab.es
}

This is an Open Access article distributed under the terms of the Creative Commons Attribution License 2.0, which permits unrestricted use, distribution, and reproduction in any medium, provided the original work is properly cited. 
Cataclysmic variables are classified as non magnetic when the magnetic field of the WD is less intense than around $10^{7}$ Gauss (10MG); then a Keplerian accretion disk forms around the WD as the result of Roche lobe overflow of the companion. Regarding magnetic CVs (white dwarf magnetic field larger than $10 \mathrm{MG}$ ), two sub-types are defined. The polars (also called AM Her stars, following the name of its representative object) have magnetic fields very intense, which prevent the formation of an accretion disk; matter falls directly onto the poles of the WD, funneled by the magnetic field. In the so-called intermediate polars (IPs, or DQ Her stars), the pressure associated to the magnetic field - less intense than in the polars - partially truncates the accretion disk, so that matter also accretes into the poles of the WD, but through an "accretion curtain" (see [26], [4] and [35] for reviews). In polars, the WD rotates synchronously with the binary orbit (due to the large magnetic field), whereas in intermediate polars the WD rotates with a period shorter than the orbital period.

The origin of nova explosions is a thermonuclear runaway, caused by the burning in degenerate conditions of the H-rich accreted matter on top of the WD. The WD envelope expands with velocities of 100's or even 1000's of km/s, leading to an increase in visual luminosity and mass ejection. Often the ejecta are enriched in CNO elements, with respect to solar abundances, as well as in Ne, showing that there has been some mixing between the accreted matter (with roughly solar composition) and the WD core (see $[12,33]$ and references therein). The chemical composition of the core is CO or $\mathrm{ONe}$, depending on the mass of the WD: $\mathrm{CO}$ for $\mathrm{M}<(1.1-1.2) \mathrm{M}_{\odot}$ and $\mathrm{ONe}$ for larger masses. A small amount of matter is ejected in nova explosions, so that a new explosion is expected once the critical mass required to reach degenerate $\mathrm{H}$-burning conditions is accreted again. Nova explosions occurring in CVs ("classical novae"), are expected to recur every $\approx 10^{4}-10^{5}$ years. The classical nova rate is around 35/yr in the Galaxy, according to [32] (see also [5]). On the other hand, there are only a handful of novae which have been recorded more than once, being their recurrence times smaller than 100 years; they are called recurrent novae. Some of these explosions are known to be hosted by symbiotic binaries, where larger (than in CVs) accretion rates are possible; it is worth noticing that the WD mass should also be quite large to enable such short recurrence periods; in addition, the WD mass is expected to grow in each eruption, and thus recurrent novae are good candidates for type Ia supernovae explosions. Less than 10 recurrent novae are known, although some novae previously classified as classical had been discovered to be recurrent in the recent years (one of them being V2487 Oph, the main topic of this paper).

Novae are expected to return to hydrostatic equilibrium after explosive ejection of a fraction of (or an amount of mass larger than) the accreted envelope. The remaining H-rich envelope (if any) burns hydrogen steadily; as its mass is depleted, the photospheric radius decreases and the effective temperature increases. The hot WD photosphere emits thermal radiation, in the super soft X-ray energy range, which can be observed once the ejecta becomes transparent enough. The duration of this super soft X-ray emitting phase depends on the nuclear burning timescale of the remaining H-rich envelope, i.e., on the amount of H-rich mass left after the explosion, which is related to the efficiency of mass-ejection during the nova explosion [28, 29, 34].

\section{X-rays from novae}

The first detections of novae in X-rays were in the 80's. The EXOSAT satellite detected the nova GQ Mus (N Mus 1983) as a soft X-ray emitter (in the interval 0.04-2 keV), 460 days after optical maximum [22]. ROSAT detected again GQ Mus (0.1-2.4 keV), 9 years after the explosion [23]. Nova Cyg 1992 was also detected by ROSAT as a bright soft X-ray source, but its emission lasted only for 18 months $[1,13]$. The luminosity in soft $\mathrm{X}$-rays was close to $\mathrm{L}_{\mathrm{Edd}}$, indicating the constancy of $\mathrm{L}_{\mathrm{bol}}$ (because L already attains such large value in the optical maximum phase) and the hardening of the 
spectrum. The turn-off times of novae deduced from soft X-ray and ultraviolet observations, ranged between 1 and 5 yr [8], except for Nova Mus 1983 (9-10 yr). Since 2004, the XRT instrument onboard Swift has monitored systematically classical novae in the super soft X-ray source phase. Much better statistics are available now, about the duration of the super soft X-ray emitting phase, and V723 Cas (Nova Cas 1995) is the record holder (more than about 15 years, see [20, 31])

In addition to soft X-rays, novae also emit harder X-rays, as observed with ROSAT [14, 17, 24] and more recently with XMM-Newton, Chandra, Suzaku and Swift. This emission originates in the ejecta, because internal and external shocks heat the expanding plasma up to temperatures of a few $\mathrm{keV}$, thus leading to the emission of X-rays with an optically thin thermal plasma spectrum (bremsstrahlung continuum plus atomic transitions lines). (Emission of very hard X-rays and VHE (very high energy) gamma-rays, as detected by Fermi/LAT for 4 novae up to now, are also related to (very strong) shocks, which can accelerate particles and lead to the production of VHE photons up to the GeV range; this is out of the scope of this paper).

Finally, X-rays emitted by post-outburst novae can also reveal the recovery of accretion in the CV, either magnetic or non magnetic. Although historically most classical novae have been classified as belonging to the class of non-magnetic CVs, in which an accretion disk around the WD can form, some novae explode in magnetic CVs, where the WD has a stronger magnetic field (see above). The supersonic accretion flow forms a shock, at some height above the WD, that heats the gas to temperatures about $10^{8} \mathrm{~K}$, for magnetic WDs. For non magnetic novae, the boundary layer between the accretion disk and the WD surface is strongly heated and emits hard X-rays.

$\mathrm{X}$-ray observations of post-outburst novae (where $\mathrm{H}$ has turned off - or not) are fundamental to study the recovery of accretion - and/or ejecta emission - tracing the properties of the CV system hosting the nova explosion. Observations of V2487 Oph (Nova Oph 1998) with XMM-Newton, have clearly revealed that accretion had been fully reestablished as soon as 2.7 years after explosion [10], perhaps indicating that accretion disks (or in general accretion streams, if the system is magnetic) are recovered fast or perhaps are not completely destroyed by the explosion.

\section{V2487 Oph = Nova Ophiuchi 1998}

V2487 Oph (Nova Oph 1998) was discovered on 1998 June 15.561 UT [19] with a maximum visual magnitude of 9.5. Spectroscopic observations three days after its outburst [6] confirmed it was a nova. Liller \& Jones [16] subsequently reported that it was a very fast nova with $t_{2} \approx 6.3$ days and $t_{3} \approx$ 9.5 days, and therefore intrinsically very bright $\left(\mathrm{t}_{n}\right.$ is the time needed to decrease in luminosity by $\mathrm{n}$ magnitudes). From near-IR observations of OI $\lambda 8446$ and $\lambda 11287$, Lynch et al. [18] established its visual extinction $A_{V}=1.16 \pm 0.24 \mathrm{mag}$, derived from the color excess $\mathrm{E}(B-V)=0.38 \pm 0.08$. Using an empirical relation between $\mathrm{t}_{2}, m_{V}^{\max }, \mathrm{A}_{V}$ and $\mathrm{E}(B-V)$, they calculated the absolute visual magnitude $M_{V}^{\max }=-8.8$ and estimated an extremely large distance, $\mathrm{d}=27 \pm 3 \mathrm{kpc}$. This would place V2487 Oph 1998 at $3.65 \mathrm{kpc}$ above the galactic plane and out of the disk, an unlikely position for this class of objects. Therefore such value for the distance must be taken with caution. However, being a fast nova, its real maximum could have been missed and probably the nova was brighter. Using the expression in Harris et al. [9] that extrapolates the visual light curve back in time to the pre-discovery data, the true $m_{V}^{\max }$ can be estimated to be in the range 6.8-9.5 mag. With these new parameters a smaller distance is obtained; the final distance to V2487 Oph 1998 ranges between 8 and $27 \mathrm{kpc}$. We will adopt $\mathrm{d}=10$ kpc. 


\subsection{X-ray observations}

We observed Nova Oph 1998 with XMM-Newton four times during 2001-2002, with an interval of 6 months between each two consecutive observations. A longer observation was made in 2007, 8.8 years after outburst. The nova was detected at all epochs, with similar spectral characteristics. In our first analysis [10], we already found that there was X-ray emission spanning from soft to hard X-rays, very different from what is expected from purely residual H-nuclear burning on the WD surface (see Fig. 1). We found that the accretion process had been reestablished already, less than $\sim 3$ years (986 days) after the explosion, as indicated by the presence of the fluorescent $\mathrm{Fe} \mathrm{K} \alpha$ emission line at 6.4 $\mathrm{keV}$; this line reveals reflection of hard X-rays on cold matter (neutral $\mathrm{Fe}$ ), most presumably from the accretion disk and/or the WD surface. A more detailed analysis of the Fe line complex shows that there are two other $\mathrm{Fe} \mathrm{K} \alpha$ emission lines, at $6.68 \mathrm{keV}$ (Fe XXV, i.e., He-like Fe) and at $6.97 \mathrm{keV}$ (Fe XXVI, i.e., H-like Fe); this Fe line complex has been observed in several CVs before, but this was the first time it was detected in a post-outburst nova. Interestingly, we also found positional correlation of V2487 Oph with the X-ray source 1RXS J173200.0-1934 detected by the RASS (ROSAT All-Sky Survey) in 1990, making V2487 Oph the first classical nova observed before and after the outburst in X-rays [10].

Observed spectra corresponding to our fourth observation (4.3 years after outburst) are displayed in Fig. 1 The best-fit model for our observations includes blackbody emission from a small fraction of the WD surface heated by the infalling matter, plus thermal plasma emission from the accretion flow material [35] (see Fig. 1 for details). The distribution of temperatures in the pre- and post-shock infalling matter is not well known, since model fitting strongly depends on the complex absorption properties. In case of partial covering [21], we get a distribution of temperatures such that the two ( $\mathrm{H}$ and He-like) Fe lines appear in a natural way. On the other hand, if we only consider a standard single absorption, the high temperature has a very large value, $\mathrm{T} \sim 70 \mathrm{keV}$ or even larger, for which $\mathrm{Fe}$ ionization would be complete and no He-like and $\mathrm{H}$-like Fe lines would appear, in contradiction with the observations. In fact, the maximum temperature of the plasma is not well constrained, since good observations at energies larger than 10-12 keV are not available. In next section we comment other observations and detections of V2487 Oph (mostly related to surveys at hard X-rays).

Timing analyses of our X-ray observations have not yielded any clear periodicity (only some hints of $\mathrm{P}_{\text {orb }} \approx 6.5$ hours, to be confirmed with optical observations).

V2487 Oph has been detected with IBIS/ISGRI in the 20-100 keV range [2, 15, 27, 30], but without good statistics. The model presented in Fig. 1 also fits such observations. In fact, INTEGRAL/IBIS has discovered many CVs of the intermediate polar sub-class; these are brighter in hard $\mathrm{X}$-rays than the CVs of the polar sub-class (see section 1), since the plasma is hotter (it is less cooled by synchrotron emission than in the polars, because the magnetic field is less strong). V2487 Oph was also detected with RXTE/PCA, showing characteristics of an intermediate polar [3]. All these observations indicate that the WD in this nova should be massive and magnetic, although the expected temporal variabilities have not been seen.

\section{Discussion and Conclusions}

A relatively recent very important discovery was that Nova Oph 1998 is a recurrent nova: a previously unknown eruption was deduced from the analysis of Harvard photographic plates from 1900 [25]. Therefore, its recurrence period is 98 years. We have tried to model such a short recurrence period: the required WD mass should be very close to the Chandrasekhar mass limit, and the accretion rate should also be very large; this is not expected in a CV, but would "require" a symbiotic system, with 


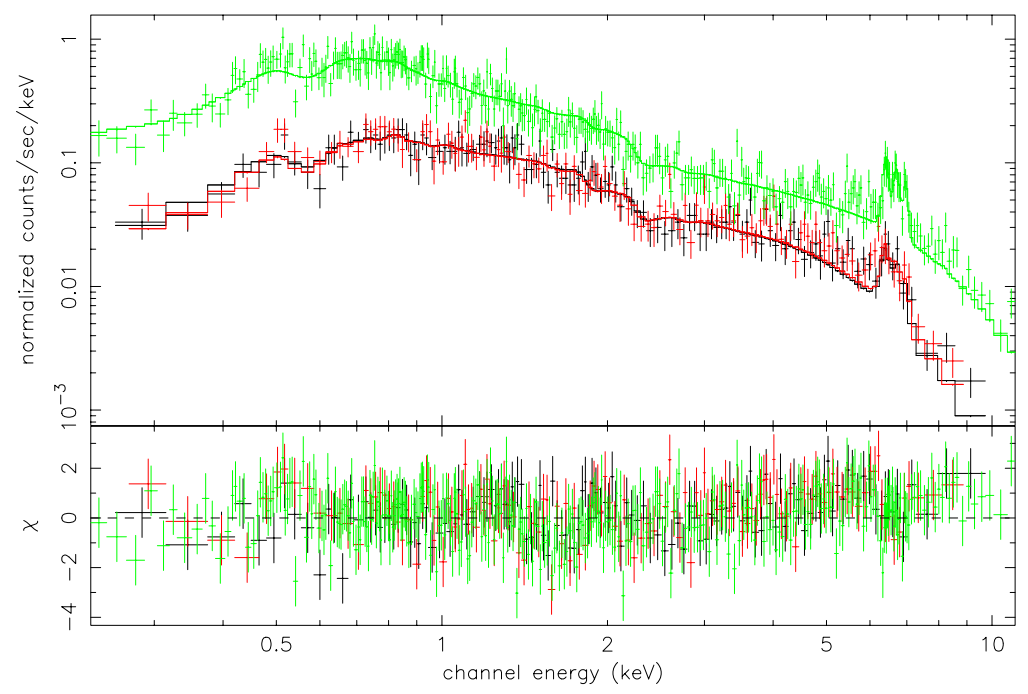

Figure 1. Observed spectrum and best-fit model of V2487 Oph, 4.3 years after outburst (upper panel) and residuals (lower panel). Data from the EPIC cameras MOS1, MOS2 and PN (black, red and green) are shown. Model components: a blackbody $\left(\mathrm{T}_{\mathrm{BB}}=120_{-30}^{+20} \mathrm{eV}, \mathrm{L}_{\mathrm{BB}, \text { bol }}=(4 \pm 1) \times 10^{34} \mathrm{erg} / \mathrm{s}\right)$, plus a $2 \mathrm{~T}$ plasma $\left(\mathrm{T}_{\text {low }}=0.3_{-0.1}^{+0.3}\right.$ and $\mathrm{T}_{\text {high }}=13_{-3}^{+8} \mathrm{keV}, \mathrm{EM}_{\text {low }}=\left(0.5_{-0.4}^{+0.6}\right) \times 10^{55}$ and $\left.\mathrm{EM}_{\text {high }}=\left(6.1_{-0.8}^{+0.9}\right) \times 10^{57} \mathrm{~cm}^{-3}\right)$, covering fraction $0.63_{-0.07}^{+0.06}$, absorbing column densities $\mathrm{N}_{\mathrm{H}}=2.1 \times 10^{21}$ and $\mathrm{N}_{\mathrm{H}}^{\mathrm{PCA}}=\left(24_{-9}^{+10}\right) \times 10^{22} \mathrm{~cm}^{-2}$, plus a gaussian line at $6.4 \mathrm{keV}$. $\mathrm{L}_{\text {unabs }}^{[0.2-10] \mathrm{keV}}=\left(8.3_{-0.4}^{+0.1}\right) \times 10^{34} \mathrm{erg} / \mathrm{s}$. Adopted distance $\mathrm{d}=10 \mathrm{kpc}$.

a red giant companion (see section 1). We are thus facing a problem to get a model for this and other recurrent novae (see [11] for models of RS Oph, a famous recurrent nova, with $\mathrm{P}_{\text {rec }}=21$ years).

Future potential X-ray missions like LOFT [7] would be crucial to solve the issue of the absence of good observations at energies larger than $10-12 \mathrm{keV}$ (see section 2). This is a very crucial point, since the mass of the WD depends on the value of the largest temperature of the thermal plasma which models its X-ray emission (if it is assumed to be equal to the accretion shock temperature). Good timing is also essential (both in X-rays and in the optical), to well determine the WD spin and the orbital period, to justify if a partial covering absorber is real or not, and to definitely classify Nova Oph 1998 as a polar or intermediate polar CV. In addition, detection of cyclotron lines in the optical is required to determine the strength of the magnetic field.

V2487 Oph is a challenging system, because it behaves like a magnetic CV (of the intermediate polar class), but at the same time it is a recurrent nova (which up to now was expected to occur in a symbiotic binary). A narrow range of possible WD masses and accretion rates is required to explain the short recurrence period of 98 years, and these are not the typical ones expected in the magnetic $\mathrm{CV}$ we see in X-rays. 
EPJ Web of Conferences

\section{Acknowledgements}

We acknowledge support from the MINECO grant AYA2011-24704.

\section{References}

[1] Balman, S., Krautter, J., Ögelman, H., ApJ 499, 395 (1998)

[2] Barlow, E.J. et al., MNRAS 372224 (2006)

[3] Butters, O. W., Norton, A. J., Mukai, K., Tomsick, J. A., A\&A 526, A77 (2011)

[4] Cropper, M., Space Sci. Rev. 54, 195 (1990)

[5] Darnley, M.J. et al., MNRAS 369257 (2006)

[6] Filippenko, A.V. et al., IAU Circular 6943 (1998)

[7] Feroci, M. et al., Exp. Astron. 34, 415 (2012)

[8] González-Riestra, R., Orio, M., Gallagher, J., A\&A 129, 23 (1998)

[9] Harris, M.J. et al. 1999, ApJ 522, 424

[10] Hernanz, M., Sala, G., Science 298, 393 (2002)

[11] Hernanz, M., José, J., New Astr. Rev. 52, 386 (2008)

[12] José, J. and Hernanz, M., ApJ, 494, 680 (1998)

[13] Krautter, J., Ögelman, H., Starrfield, S., Wichmann, R., Pfeffermann, E., ApJ 456, 788 (1996)

[14] Krautter, J., in Classical Nova Explosions, eds. M. Hernanz and J. José (AIP CP 637, New York, 2002), p. 345

[15] Krivonos, R. et al., A\&A 523, A61 (2010)

[16] Liller W., Jones, A.F., IBVS 4774, 1 (1999)

[17] Lloyd, H.M. et al., Nature, 356, 222 (1992)

[18] Lynch, D.K. et al. 2000, ApJ, 541, 791

[19] Nakano, S. et al., IAUC \#6941 (1998)

[20] Ness, J.-U. et al., ApJ 663, 505 (2007)

[21] Norton, A.J., Watson, M.G., MNRAS 237, 853 (1989)

[22] Ögelman, H., Beuermann, K. and Krautter, J., ApJ 287, L31 (1984)

[23] Ögelman, H., Orio, M., Krautter, J., Starrfield, S., Nature 361, 331 (1993)

[24] Orio, M., Covington, J., Ögelman, H., A\&A 373, 542 (2001)

[25] Pagnotta, A., Schaefer, B.E., Xiao, L., Collazzi, SA.C., Kroll, P., AJ 138, 1230 (2009)

[26] Patterson, J.P., PASP 106, 209 (1994)

[27] Revnivtsev, M., Sazonov, S., Krivonos, R., Ritter, H., Sunyaev, R., A\&A 489, 1121 (2008)

[28] Sala, G., Hernanz, M., A\&A 439, 1057 (2005)

[29] Sala, G., Hernanz, M., A\&A 439, 1061 (2005)

[30] Scaringi, S. et al., MNRAS 401, 2207 (2010)

[31] Schwarz, G.J. et al., ApJS 197, 31 (2011)

[32] Shafter, A.W. (2002), in Classical Nova Explosions, eds. M. Hernanz and J. José, (AIP CP 637, New York, 2002), p. 462

[33] Starrfield, S., Truran, J.W., Wiescher, M., Sparks, W.M., MNRAS 296, 502 (1998)

[34] Tuchman, Y., Truran, J.W., ApJ 503, 381 (1998)

[35] Wu, K., Space Sci. Rev. 93, 611 (2000) 